\title{
Higher levels of stress and different coping strategies are associated with greater morning and evening fatigue severity in oncology patients receiving chemotherapy
}

\author{
Fay Wright ${ }^{1} \cdot$ Kord M. Kober ${ }^{2}$ - Bruce A. Cooper ${ }^{2}$ - Steven M. Paul ${ }^{2}$ - Yvette P. Conley ${ }^{3}$ - Marilyn Hammer ${ }^{4}$. \\ Jon D. Levine ${ }^{5}$. Christine Miaskowski ${ }^{2,5,6}$ iD
}

Received: 20 September 2019 / Accepted: 9 January 2020 / Published online: 20 January 2020

(C) Springer-Verlag GmbH Germany, part of Springer Nature 2020

\begin{abstract}
Purpose A cancer diagnosis and associated treatments are stressful experiences for most patients. Patients' perceptions of stress and their use of coping strategies may influence fatigue severity. This study extends our previous work describing distinct profiles of morning (i.e., Very Low, Low, High, and Very High) and evening (i.e., Low, Moderate, High, and Very High) fatigue in oncology patients by evaluating for differences in stress and coping strategies among these fatigue classes.

Methods This longitudinal study evaluated for changes in morning and evening fatigue in oncology patients $(n=1332)$ over two cycles of chemotherapy (CTX). Patients completed measures of cumulative exposure to stressful life events (SLEs) (i.e., the Life Stressor Checklist-Revised), general stress (i.e., Perceived Stress Scale [PSS]), cancer-specific stress (i.e., Impact of Event ScaleRevised [IES-R]), and coping strategies (i.e., Brief Cope). Differences among the latent classes were evaluated using analyses of variance, Kruskal-Wallis, or chi-square tests.

Results Patients in both the Very High morning and evening fatigue classes reported higher numbers of and a higher impact from previous SLEs and higher PSS scores than the other fatigue classes. The IES-R scores for the Very High morning fatigue class met the criterion for subsyndromal PTSD. Patients in the Very High evening fatigue class used a higher number of engagement coping strategies compared with the Very High morning fatigue class.

Conclusions Our findings suggest that interventions to reduce stress and enhance coping warrant investigation to decrease fatigue in patients undergoing CTX.
\end{abstract}

Keywords Morning fatigue $\cdot$ Evening fatigue $\cdot$ Chemotherapy $\cdot$ Cumulative life stress $\cdot$ Coping $\cdot$ Stress $\cdot$ Cancer

Electronic supplementary material The online version of this article (https://doi.org/10.1007/s00520-020-05303-5) contains supplementary material, which is available to authorized users.

Christine Miaskowski

chris.miaskowski@ucsf.edu

1 Rory Meyers College of Nursing, New York University, New York, NY, USA

2 School of Nursing, University of California, San Francisco, CA, USA

3 School of Nursing, University of Pittsburgh, Pittsburgh, PA, USA

4 Dana Farber Cancer Center, Boston, MA, USA

5 School of Medicine, University of California, San Francisco, CA, USA

6 Department of Physiological Nursing, University of California, 2 Koret Way - N631F, San Francisco, CA 94143-0610, USA

\section{Introduction}

A cancer diagnosis and associated treatments are stressful experiences for most patients [1]. Stress initiates a cascade of pathways in the peripheral and central nervous systems that activates the autonomic nervous system (ANS) and/or the hypothalamic-pituitary-adrenal (HPA) axis to maintain homeostasis [2]. Cognitive and emotional feedbacks from the cortical and limbic areas of the brain modulate the activity of hypothalamic and brain stem structures directly controlling ANS and HPA activity [2, 3]. Fatigue is an adaptive response to acute stress that conserves energy and maintains homeostasis [3]. With repeated stressful events or cumulative exposure to stressful life events (SLEs), the ANS and HPA experience increased allostatic load resulting in increased fatigue severity [4]. 
Fatigue is the most common and debilitating symptom experienced by oncology patients during chemotherapy (CTX). Inter-individual variability in fatigue severity is influenced by demographic, clinical, psychological, behavioral, and biological characteristics [5]. In prior work [6, 7], we described four classes of oncology patients $(N=$ 1332) with distinct profiles of morning and evening fatigue severity, and demographic, clinical, and co-occurring symptom characteristics that distinguished among these classes. Using clinically meaningful cutoff scores for the Lee Fatigue Scale (i.e., 3.2 for morning and 5.6 for evening), we labeled the four distinct morning (i.e., Very Low, Low, High, and Very High) [6] and four distinct evening (i.e., Low, Moderate, High, and Very High) [7] fatigue profiles derived using latent profile analysis (LPA; see Supplemental Figure 1).

Patients' perceptions of stress and their use of coping strategies may influence fatigue severity during CTX. Differences in the perception and evaluation of external events create variability in ANS and HPA activity levels, resulting in variable levels of perceived stress [3] that may influence inter-individual variability in fatigue severity. While higher levels of perceived stress were associated with greater fatigue severity [8-15], the majority of these studies were cross-sectional and evaluated only women with breast cancer [8-10, 13-15].

Coping encompasses a broad range of cognitive and behavioral responses to stress to maintain homeostasis [16]. Patients can use multiple coping strategies based on their appraisal of the internal (e.g., acceptance) and external (e.g., social support systems) resources available to them to reduce, master, or tolerate stress [16]. In addition, lifetime exposure to stressful experiences (e.g., physical or sexual assault) and current life stressors (e.g., cancer diagnosis and treatment) influence coping [16]. Coping is often described in terms of the use of engagement (e.g., actively doing something to alter the stressor) or disengagement (e.g., avoidance) strategies [16-18]. In the four studies that examined the association between coping and fatigue during CTX, greater fatigue severity was associated with disengagement coping $[19,20]$, while lower fatigue severity was associated with engagement coping [21, 22], However, these studies did not examine the impact of cumulative exposure to SLEs on coping and fatigue. One study found that increased exposure to SLEs was associated with greater fatigue severity in women with breast cancer; however, the effects of coping were not evaluated [23]. Given the paucity of research on the relationships between stress and coping and fatigue, the purpose of this study was to evaluate for differences in cumulative exposure to SLEs, general stress, cancer-specific stress, and coping strategies among our previously identified morning [6] and evening [7] fatigue classes.

\section{Methods}

\section{Patients and settings}

These analyses used data from a longitudinal study that evaluated the symptom experience of oncology patients receiving CTX [24]. Written informed consent was obtained from English literate patients $\geq 18$ years of age who had received one or two cycles of CTX for a diagnosis of breast, gastrointestinal, gynecological, or lung cancer within the 4 weeks prior to enrollment and were scheduled for at least two additional CTX cycles. Patients were recruited from seven different sites that included community-based oncology programs and Comprehensive Cancer Centers. Of the 2234 patients approached, 1343 consented to participate $(60.1 \%$ response rate). Patients' main reason for refusal was feeling overwhelmed with their CTX treatment. Additional methodological details are described elsewhere [6, 7].

\section{Instruments}

\section{Fatigue measures}

The 18-item Lee Fatigue Scale (LFS) is designed to assess physical fatigue and energy [25]. Each item was rated on a 0 to 10 numeric rating scale (NRS). Fatigue scores were calculated as the mean of the 13 fatigue items. Higher scores indicate greater fatigue severity. Using separate LFS questionnaires, patients rated each item based on how they felt within 30 min of awakening (i.e., morning fatigue) and prior to going to bed (i.e., evening fatigue). The LFS has established cutoff scores for clinically meaningful levels of fatigue (i.e., $\geq 3.2$ for morning fatigue, $\geq 5$.6 for evening fatigue) [26]. The LFS has well-established validity and reliability and is easy to administer [25]. In our study, Cronbach's alphas were 0.96 for morning and 0.93 for evening fatigue.

\section{General stress measures}

The Life Stressor Checklist-Revised (LSC-R) is a 30 -item inventory of lifetime exposure to stressful, potentially traumatic events (e.g., physical assault, death of a loved one) [27]. The total LSC-R score was obtained by summing the number of events endorsed (possible range is $0-30$, with 30 indicating that the patient experienced all of the events). If the patient endorsed an event, they indicated how much that stressor affected their life in the past year, using a 1 ("not at all") to 5 ("extremely") scale. These responses were averaged to yield a mean "affected" score. The LSC-R has good to moderate test-retest reliability and criterion-related validity in diverse populations [28].

The 14-item Perceived Stress Scale (PSS) is a measure of perceived stress according to the degree that life 
circumstances are appraised as stressful over the previous 4 weeks [29]. Each item was rated on a 0 ("never") to 4 ("very often") scale. Total scores were summed after reversing the seven positive items' scores and can range from 0 to 56 . A higher score indicates greater stress. The PSS has wellestablished validity and reliability [29]. In our study, its Cronbach's alpha was 0.85 .

\section{Cancer-specific stress measure}

The 22-item Impact of Event Scale-Revised (IES-R) is used to measure cancer-related stress [30]. Patients rated each item based on how stressful each potential event was for them in the past week on a 0 (not at all) to 4 (extremely) scale. Three subscale (e.g., intrusion, avoidance, and hyperarousal) scores and a total score were calculated [30]. The total score can range from 0 to 88 . A clinical cutoff score of 33 represents probable post-traumatic stress disorder (PTSD), and scores of $\geq 37$ suggest high levels of post-traumatic symptoms [30]. The IES-R has well-established validity and reliability [30]. In this sample, its Cronbach's alpha was 0.91 .

\section{Coping strategy measure}

The 28-item Brief Cope scale measures patients' use of various engagement and disengagement coping strategies [31]. For each strategy, patients rated the level of strategy use using a 4-point Likert scale that ranged from 1 ("I haven't been doing this at all") to 4 ("I have been doing this a lot"). Higher scores indicate greater use of each coping strategy. Fourteen total dimensions, represented by two items each, were evaluated using this instrument (with their respective Cronbach's alphas): self-distraction (0.46), active coping (0.75), denial (0.72), substance use (0.87), use of emotional support (0.77), use of instrumental support (0.77), behavioral disengagement $(0.57)$, venting $(0.65)$, positive reframing (0.79), planning $(0.74)$, humor $(0.83)$, acceptance $(0.68)$, religion (0.92), and self-blame (0.73). The Brief Cope has wellestablished validity and reliability in oncology patients [32].

\section{Study procedures}

The study was approved by the Institutional Review Board at each study site. A research team member approached eligible patients during their first or second CTX cycle to discuss participation and obtain written informed consent. Depending on the length of their CTX cycle, patients completed the fatigue questionnaires in their homes, a total of six times over two CTX cycles (i.e., before CTX administration, approximately 1 week after CTX administration, approximately 2 weeks after CTX administration). Due to the potentially sensitive nature of LSC-R items, patients were given three options for its completion: in person with a research team member, over the telephone, or on their own. Patients were reminded that they could refuse to answer questions that caused discomfort.

\section{Data analysis}

Data were analyzed using SPSS version 22 (IBM, Armonk, NY). Descriptive statistics and frequency distributions were calculated for the stress and coping measures. As previously reported [6, 7], LPA was used to identify patient subgroups with distinct morning or evening fatigue profiles over the six assessments. We used the identified profiles in these analyses. Differences in general and cancer-specific stress scores and coping strategies scores among the classes were evaluated using analyses of variance, Kruskal-Wallis, or chi-square tests with Bonferroni-corrected post hoc contrasts (i.e., $p<.008$ $[0.05 / 6])$.

\section{Results}

\section{Sample characteristics}

In terms of differences in demographic and clinical characteristics among the latent classes [6,7], in brief, the overall sample was predominantly female, white, and college educated. Compared with the other fatigue classes, patients in both the Very High morning and evening fatigue classes were younger and reported a higher comorbidity burden. Patients in the Very High morning fatigue class were more likely to live alone and had a higher body mass index. Patients in the Very High evening fatigue class were more likely to be female, have childcare responsibilities, and have a lower functional status.

\section{Morning fatigue}

\section{Differences in cumulative life stress and general stress}

In terms of cumulative life stress, significant differences were found among the morning fatigue classes for both the number and impact of SLEs (Table 1). Compared with the Very Low and Low classes (i.e., 5.2 and 5.4, respectively), patients in the High and Very High classes reported a higher number of SLEs (i.e., 6.4 and 8.5, respectively). An identical pattern of differences in the impact of SLEs was found among the morning fatigue classes. Compared with the High class, patients in the Very High class reported a higher number and a higher impact of SLEs. In terms of general stress, across the four classes, as the profiles of morning fatigue severity increased, PSS scores increased (i.e., Very Low < Low < High < Very High; Table 1). 


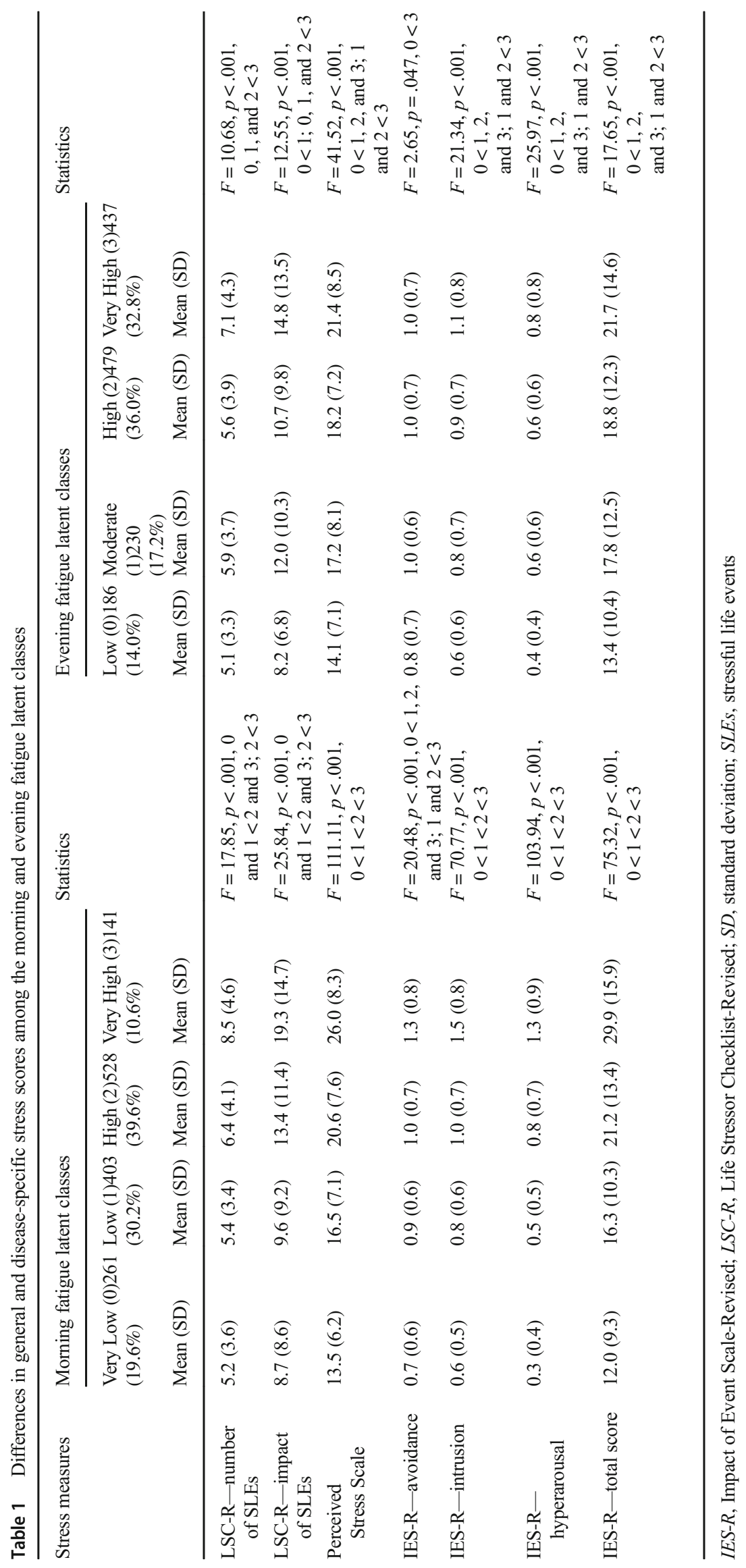




\section{Differences in cancer-specific stress}

For the intrusion and hyperarousal subscale and total scores of the IES-R, the post hoc contrasts were significant in the following pattern: Very Low $<$ Low $<$ High $<$ Very High. For the avoidance subscale, compared with the Very Low class, patients in the Low, High, and Very High classes reported higher scores (Table 1).

\section{Differences in coping}

Significant differences were found among the morning fatigue classes in the frequency of use of eight of the fourteen (57\%) coping strategies (Table 2). Compared with the Very Low class, patients in the Very High class were less likely to use active coping and more likely to use denial. Compared with the Very Low class, patients in the High class were less likely to use acceptance. Compared with the Low class, patients in the High class were more likely to use instrumental support. Compared with the Very Low class, patients in the Low, High, and Very High classes were more likely to use self-distraction. Compared with the Very Low class, patients in the High and Very High classes were more likely to use behavioral disengagement. Compared with the Very Low, Low, and High classes, patients in the Very High class were more likely to use venting. Compared with the Very Low and Low classes, patients in the High and Very High classes were more likely to use self-blame. No significant differences were found among the morning fatigue classes in the use of planning, positive reframing, humor, religion, emotional support, and substance use.

\section{Evening fatigue}

\section{Differences in cumulative life stress and general stress}

In terms of cumulative life stress, compared with the Low, Moderate, and High classes, patients in the Very High class reported a higher number of SLEs (i.e., 5.1, 5.9, 5.6, and 7.1, respectively; Table 1). In terms of the impact of SLEs, compared with the Low, Moderate, and High classes, patients in the Very High class reported higher SLE impact scores (i.e., 8.2, 12.0, 10.7, and 14.8, respectively; Table 1). In addition, compared with the Low class, patients in the Moderate class reported higher SLE impact scores. In terms of general stress, compared with the Low class, patients in the other three evening fatigue classes reported higher PSS scores. In addition, compared with the Moderate and High classes, patients in the Very High class reported higher PSS scores (Table 1).
Differences in cancer-specific stress

Compared with the Low class, patients in the Very High class reported higher IES-R avoidance scores. Compared with the Low class, patients in the Moderate, High, and Very High classes reported higher intrusion, hyperarousal, and IES-R total scores. In addition, compared with the Moderate and High classes, patients in the Very High class reported higher intrusion, hyperarousal, and IES-R total scores (Table 1).

\section{Differences in coping}

Significant differences were found among the evening fatigue classes in the frequency of use of eight of the fourteen (57\%) coping strategies (Table 2). Compared with patients in the other three classes, patients in the Very High class were more likely to use planning, emotional support, instrumental support, and self-blame. Compared with the Low class, patients in the High and Very High classes were more likely to use humor and self-distraction. Compared with the High and Very High classes, patients in the Moderate class were more likely to use religion. Compared with the Low and Moderate classes, patients in the High and Very High classes were more likely to use venting. Compared with the other three classes, patients in the Very High class were more likely to use self-blame. No significant differences were found among the evening fatigue classes in the use of active coping, positive reframing, acceptance, denial, substance use, and behavioral disengagement.

\section{Discussion}

This study extends our previous work identifying patients with morning [6] and evening [7] fatigue profiles to include associations with cumulative life stress, cancer-specific stress, and coping. While previous research found associations between childhood SLEs and increased risk for greater fatigue severity in women with breast cancer [8], our study is the first to evaluate the impact of SLEs on fatigue severity in a sample of patients with heterogeneous cancer diagnoses. Compared with the other three classes, patients in both the Very High morning and evening fatigue classes reported higher numbers of and higher impact from previous SLEs. The increased allostatic load associated with cumulative SLEs [4] is a plausible explanation for these associations. For example, higher levels of and higher impact from SLEs increased the risk of experiencing chronic pain [33], as well as fatigue and depression [34]. These findings suggest that SLEs in oncology patients may be associated with increased risk for multiple cooccurring symptoms and warrant further study.

Given that higher cumulative life stress was associated with increased allostatic load in healthy adults and in patients with irritable bowel syndrome [35], our findings of higher PSS 


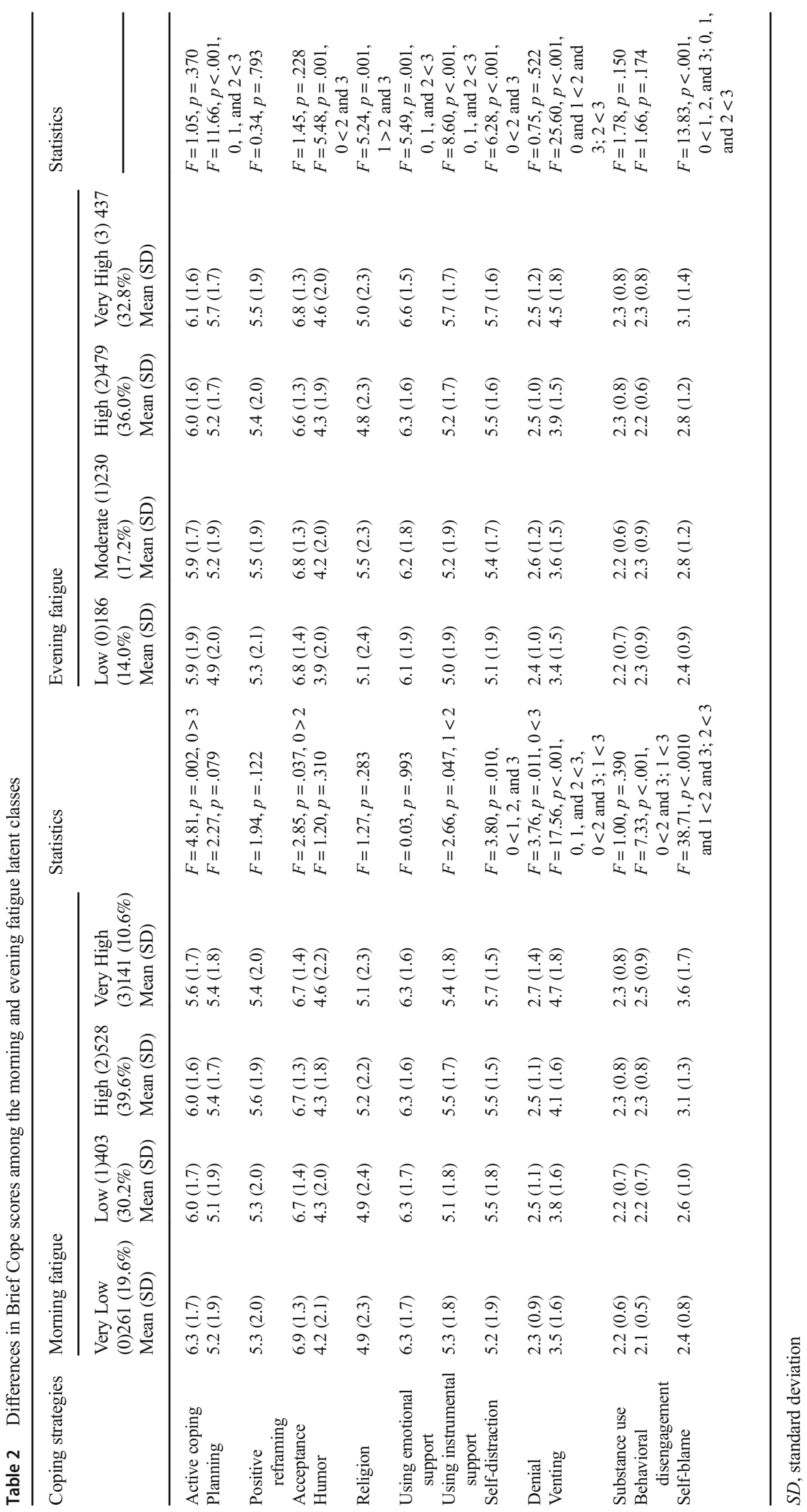


Table 3 Comparison of the stress measures and coping strategies associated with membership in the highest morning and evening fatigue classes compared with the lowest morning and evening fatigue classes

\begin{tabular}{|c|c|c|}
\hline Characteristics & Very High morning fatigue & Very High evening fatigue \\
\hline \multicolumn{3}{|l|}{ Stress measures } \\
\hline LSC-R—number of SLEs & $\uparrow$ & $\uparrow$ \\
\hline LSC-R_impact of SLEs & $\uparrow$ & $\uparrow$ \\
\hline Perceived Stress Scale & $\uparrow$ & $\uparrow$ \\
\hline IES-R—avoidance & $\uparrow$ & $\uparrow$ \\
\hline IES-R - intrusion & $\uparrow$ & $\uparrow$ \\
\hline IES-R—hyperarousal & $\uparrow$ & $\uparrow$ \\
\hline IES-R-total score & $\uparrow$ & $\uparrow$ \\
\hline \multicolumn{3}{|l|}{ Coping strategies } \\
\hline \multicolumn{3}{|l|}{ Engagement } \\
\hline Active coping & $\downarrow$ & \\
\hline Planning & & $\uparrow$ \\
\hline Using emotional support & & $\uparrow$ \\
\hline Using instrumental support & & $\uparrow$ \\
\hline \multicolumn{3}{|l|}{ Disengagement } \\
\hline Avoidance & $\uparrow$ & \\
\hline Denial & $\uparrow$ & \\
\hline Self-blame & $\uparrow$ & $\uparrow$ \\
\hline \multicolumn{3}{|l|}{ Emotion-based } \\
\hline Venting & $\uparrow$ & $\uparrow$ \\
\hline \multicolumn{3}{|l|}{ Other subscales } \\
\hline Self-distraction & $\uparrow$ & $\uparrow$ \\
\hline Humor & & $\uparrow$ \\
\hline Religion & & $\downarrow$ \\
\hline
\end{tabular}

$\uparrow=$ Higher score for the highest morning and evening fatigue classes compared with the lowest classes $\downarrow=$ Lower score for the highest morning and evening fatigue classes compared with the lowest classes IES-R, Impact of Event Scale-Revised; LSC-R, Life Stressor Checklist-Revised; SLEs, stressful life events

The coping strategies of positive reframing, acceptance, religion, and substance use are not included in the table because no significant differences were found between the highest morning and evening fatigue latent classes compared with the lowest classes scores in the Very High morning and evening fatigue classes, compared with the other classes, suggests that this mechanism may occur in oncology patients. Equally important, PSS scores were associated with greater fatigue severity in adults with rheumatoid arthritis, fibromyalgia, and inflammatory bowel disease [36] and unexplained chronic fatigue [37]. Because these conditions and fatigue associated with cancer share inflammation as a common underlying mechanism, future studies need to examine the additive or synergistic effects of stress and inflammatory mechanisms on fatigue severity.

A cancer diagnosis and its treatment(s) are significant stressors, with $20 \%$ of patients reporting cancer-related subsyndromal PTSD [1]. The IES-R total score for patients in the Very High morning fatigue class (i.e., 29.9) meets the criterion for subsyndromal PTSD [30]. This finding is consistent with previous reports of greater fatigue severity being associated with higher IES-R total scores in patients with Cushing's disease [38] and survivors of Middle East respiratory syndrome [39]. While the patients in the Very High evening fatigue class had IES-R total scores below the clinically meaningful cutoff for subsyndromal PTSD (i.e., IES-R total score of 21.7), their scores were significantly higher than those of the other three evening fatigue classes, which suggests that cancer-specific stress is a risk factor for greater evening fatigue severity. Given the negative impact of fatigue on oncology patients receiving CTX [40], the higher scores on the avoidance, intrusion, and hyperarousal subscales of the IES-R are not unexpected. Our findings suggest that interventions to decrease cancer-specific stress (e.g., yoga [41]) may decrease fatigue severity.

In terms of the impact of coping strategies on fatigue, in older oncology patients (i.e., > 70 years), disengagement coping strategies were associated with higher fatigue severity [42]. In contrast, engagement coping strategies mediated fatigue's impact during [42] and after [42, 43] cancer treatment completion. In studies that explored the perceptions of effective coping strategies for fatigue in patients with chronic obstructive pulmonary disease [43] and end-stage kidney disease 
(ESKD) [44], both groups valued clinicians' acknowledgment of fatigue variability, support to deal with the impact of fatigue in their daily lives, and active participation in individualizing interventions [43, 44].

Noticeable similarities and differences were apparent in the coping strategies used by patients in the Very High morning and Very High evening fatigue classes (Table 3). Patients in the Very High evening fatigue class identified using engagement coping strategies more frequently than patients in the Very High morning fatigue class. One possible explanation for this difference in the use of engagement coping strategies may be the impact of morning compared with evening fatigue. In healthy adults, awakening refreshed without fatigue is considered "normal" [45]. Therefore, a patient may not actively plan or seek support when they expect to feel less fatigued in the morning. Educating patients about diurnal variability in fatigue and its potential impact on daily activities may increase the use of engagement coping strategies.

The finding that both the Very High morning and evening fatigue groups used self-blame as a coping strategy is consistent with prior research that identified self-blame as a predictor of fatigue in new parents [46], as well as in patients with melanoma [47], multiple sclerosis [48], and breast cancer [49]. In contrast, self-compassion was identified by patients with ESKD as an effective intervention to decrease their selfblame when they needed to ask for support to cope with their fatigue [44]. Teaching self-compassion may support patients to use more engaged coping behaviors for their stress and fatigue.

As an emotion-based coping strategy, venting refers to focusing on and expressing negative feelings [17]. While not evaluated in oncology patients, venting was found to increase patients' ability to cope with both positive and negative feelings about multiple sclerosis [48] and may warrant evaluation as a strategy for fatigue.

Our finding that patients in both the Very High morning and evening fatigue classes used self-distraction to cope with fatigue is not unexpected. Self-distraction was identified as an effective coping strategy for fatigue in oncology patients during CTX [20], in patients with ESKD [44], and in healthy working adults [50]. Self-distraction may be used to shift the focus from fatigue without eliminating or treating the symptom [50].

While using humor as a coping strategy was associated with decreased stress in women with breast cancer [51], it was not associated with decreased fatigue severity [49]. In general, humor is a personal experience, used in different ways and situations based on culture and gender [52]. Therefore, it is possible that the patients' personalities in the Very High evening fatigue class may have influenced their use of humor. The influence of personality characteristics on the use of various coping strategies to decrease fatigue warrants additional investigation.
Religion and spirituality have distinct and overlapping constructs [53]. The religion subscale of the Brief Cope assesses several of these overlapping constructs (e.g., using prayer or meditation) [31]. In oncology patients, spiritual well-being was associated with lower fatigue severity [54]. However, religious/spiritual coping support is not a consistent part of patient care [53]. Assessing patients' interest in the use of religious/spiritual coping strategies and providing support for these strategies may assist with fatigue management. Further research on the association between fatigue and religious/spiritual coping strategies is warranted.

Study limitations should be acknowledged. Patients were recruited at various times during their cancer treatment. Therefore, cancer-specific stress at diagnosis was not evaluated. Because the LSC-R is a retrospective evaluation, it is possible that the patients' current symptom experiences or cancer diagnosis and treatment influenced their memories of past events. While the subjective measures of stress are valid and reliable, future studies should examine associations between the severity of morning and evening fatigue and objective measures of stress (e.g., hair cortisol) as well as inflammatory genes and their expression and regulation. In addition, future studies should examine the extreme fatigue classes (i.e., Very Low morning versus Low evening, and Very High morning versus Very High evening) for differences in stress and coping strategies.

Despite these limitations, this longitudinal study with a large representative sample of oncology patients is the first to evaluate for associations among SLEs, general stress, cancer-specific stress, and the use various coping strategies and greater morning and evening fatigue severity during CTX. In addition, prior research has identified that higher levels of SLEs may decrease a patient's participation in health-related behaviors (e.g., physical activity) [55], which may affect adherence to exercise interventions prescribed to decrease fatigue severity. Additional studies are needed that evaluate the effect of stress management strategies and interventions targeted at the use of disengagement coping strategies to mitigate morning and evening fatigue.

Author's contributions Conceptualization and methodology: FW, KMK, $\mathrm{BAC}, \mathrm{SMP}$, and CM

Data collection: $\mathrm{FW}, \mathrm{MH}$, and $\mathrm{CM}$

Original draft preparation: FW, KMK, and CM

Review and editing: all of the authors

Approval of the final manuscript: all of the authors

Funding information This study was supported by a grant from the National Cancer Institute (NCI, CA134900). Dr. Miaskowski is an American Cancer Society Clinical Research Professor and is funded by a K05 award from the NCI (CA168960).

Data availability The authors had full control of all of the primary data and will allow the journal to review the data if requested to do so. 


\section{Compliance with ethical standards}

The study was approved by the Institutional Review Board at each study site. A research team member approached eligible patients during their first or second CTX cycle to discuss participation and obtain written informed consent.

Conflict of interest The authors declare that they have no conflicts of interest.

\section{References}

1. Cordova MJ, Riba MB, Spiegel D (2017) Post-traumatic stress disorder and cancer. Lancet Psychiatry 4(4):330-338. https://doi. org/10.1016/S2215-0366(17)30014-7

2. Chrousos GP, Gold PW (1992) The concepts of stress and stress system disorders. Overview of physical and behavioral homeostasis. JAMA 267(9):1244-1252

3. Kop WJ, Kupper HM (2016) Fatigue and stress ¿s. In: Fink G (ed) Stress: concepts, cognition, emotion, and behavior. Academic Press, San Diego, pp 345-350. https://doi.org/10.1016/b978-0-12800951-2.00043-1

4. Shields GS, Slavich GM (2017) Lifetime stress exposure and health: a review of contemporary assessment methods and biological mechanisms. Soc Personal Psychol Compass 11(8):e12335. https://doi.org/10.1111/spc3.12335

5. Saligan LN, Olson K, Filler K, Larkin D, Cramp F, Yennurajalingam S, Escalante CP, del Giglio A, Kober KM, Kamath J, Palesh O, Mustian K, Multinational Association of Supportive Care in Cancer Fatigue Study Group-Biomarker Working Group (2015) The biology of cancer-related fatigue: a review of the literature. Support Care Cancer 23(8):2461-2478. https://doi.org/10.1007/s00520-015-2763-0

6. Wright F, Dunn LB, Paul SM, Conley YP, Levine JD, Hammer MJ, Cooper BA, Miaskowski C, Kober KM (2019) Morning fatigue severity profiles in oncology outpatients receiving chemotherapy. Cancer Nurs 42(5):355-364. https://doi.org/10.1097/NCC. 0000000000000626

7. Wright F, Cooper BA, Conley YP, Hammer MJ, Chen LM, Paul SM, Levine JD, Miaskowski C, Kober KM (2017) Distinct evening fatigue profiles in oncology outpatients receiving chemotherapy. Fatigue 5(3):131-144. https://doi.org/10.1080/21641846.2017. 1322233

8. Bower JE, Asher A, Garet D, Petersen L, Ganz PA, Irwin MR, Cole SW, Hurvitz SA, Crespi CM (2019) Testing a biobehavioral model of fatigue before adjuvant therapy in women with breast cancer. Cancer 125(4):633-641. https://doi.org/10.1002/cncr.31827

9. Yee MK, Sereika SM, Bender CM, Brufsky AM, Connolly MC, Rosenzweig MQ (2017) Symptom incidence, distress, cancerrelated distress, and adherence to chemotherapy among African American women with breast cancer. Cancer 123(11):2061-2069. https://doi.org/10.1002/cncr.30575

10. Reinertsen KV, Engebraaten O, Loge JH, Cvancarova M, Naume B, Wist E, Edvardsen H, Wille E, Bjoro T, Kiserud CE (2017) Fatigue during and after breast cancer therapy - a prospective study. J Pain Symptom Manag 53(3):551-560. https://doi.org/10.1016/j. jpainsymman.2016.09.011

11. Araujo JKL, Giglio AD, Munhoz BA, Fonseca FLA, Cruz FM, Giglio AD (2017) Chemotherapy-induced fatigue correlates with higher fatigue scores before treatment. Am J Hosp Palliat Care 34(5):404-411. https://doi.org/10.1177/1049909116629134

12. Sakamoto N, Takiguchi S, Komatsu H, Okuyama T, Nakaguchi T, Kubota Y, Ito Y, Sugano K, Wada M, Akechi T (2017) Supportive care needs and psychological distress and/or quality of life in ambulatory advanced colorectal cancer patients receiving chemotherapy: a cross-sectional study. Jpn J Clin Oncol 47(12):1157-1161. https://doi.org/10.1093/jjco/hyx152

13. Abrahams HJG, Gielissen MFM, de Lugt M, Kleijer EFW, de Roos WK, Balk E, Verhagen C, Knoop H (2017) The Distress Thermometer for screening for severe fatigue in newly diagnosed breast and colorectal cancer patients. Psycho-oncology 26(5):693697. https://doi.org/10.1002/pon.4208

14. Von Ah DM, Kang DH, Carpenter JS (2008) Predictors of cancerrelated fatigue in women with breast cancer before, during, and after adjuvant therapy. Cancer Nurs 31(2):134-144. https://doi.org/10. 1097/01.NCC.0000305704.84164.54

15. Ho RT, Kwan TT, Cheung IK, Chan CK, Lo PH, Yip PS, Luk MY, Chan CL (2015) Association of fatigue with perceived stress in Chinese women with early stage breast cancer awaiting adjuvant radiotherapy. Stress Health 31(3):214-221. https://doi.org/10.1002/ smi.2548

16. Folkman S, Lazarus RS, Gruen RJ, DeLongis A (1986) Appraisal, coping, health status, and psychological symptoms. J Pers Soc Psychol 50(3):571-579. https://doi.org/10.1037//0022-3514.50.3. 571

17. Carver CS, Scheier MF, Weintraub JK (1989) Assessing coping strategies: a theoretically based approach. J Pers Soc Psychol 56(2):267-283

18. Langford DJ, Cooper B, Paul S, Humphreys J, Keagy C, Conley YP, Hammer MJ, Levine JD, Wright F, Melisko M, Miaskowski C, Dunn LB (2017) Evaluation of coping as a mediator of the relationship between stressful life events and cancer-related distress. Health Psychol 36(12):1147-1160. https://doi.org/10.1037/hea0000524

19. Ahlberg K, Ekman T, Wallgren A, Gaston-Johansson F (2004) Fatigue, psychological distress, coping and quality of life in patients with uterine cancer. J Adv Nurs 45(2):205-213

20. Dahal A, Meheta RK (2018) Fatigue experience and coping strategies among cancer patients receiving chemotherapy. J Nepal Health Res Counc 16(3):285-290

21. Dsouza A, Kamboj R, Mandavkar S, Chavan N, Ramaswamy A, Ostwal V (2018) An evaluation of early-onset fatigue and the related coping strategies in patients with gastrointestinal cancer: a prospective pilot study. Indian J Cancer 55(2):162-165. https://doi.org/ 10.4103/ijc.IJC_568_17

22. Reuter K, Classen CC, Roscoe JA, Morrow GR, Kirshner JJ, Rosenbluth R, Flynn PJ, Shedlock K, Spiegel D (2006) Association of coping style, pain, age and depression with fatigue in women with primary breast cancer. Psycho-oncology 15(9):772779. https://doi.org/10.1002/pon.1012

23. Bower JE, Crosswell AD, Slavich GM (2014) Childhood adversity and cumulative life stress: risk factors for cancer-related fatigue. Clin Psychol Sci 2(1):108-115. https://doi.org/10.1177/ 2167702613496243

24. Miaskowski C, Cooper BA, Aouizerat B, Melisko M, Chen LM, Dunn L, Hu X, Kober KM, Mastick J, Levine JD, Hammer M, Wright F, Harris J, Armes J, Furlong E, Fox P, Ream E, Maguire R, Kearney N (2017) The symptom phenotype of oncology outpatients remains relatively stable from prior to through 1 week following chemotherapy. Eur J Cancer Care 26(3). doi:https://doi.org/10. 1111/ecc. 12437

25. Lee KA, Hicks G, Nino-Murcia G (1991) Validity and reliability of a scale to assess fatigue. Psychiatry Res 36(3):291-298

26. Fletcher BS, Paul SM, Dodd MJ, Schumacher K, West C, Cooper B, Lee K, Aouizerat B, Swift P, Wara W, Miaskowski CA (2008) Prevalence, severity, and impact of symptoms on female family caregivers of patients at the initiation of radiation therapy for prostate cancer. J Clin Oncol 26(4):599-605. https://doi.org/10.1200/ JCO.2007.12.2838 
27. Asgeirsdottir HG, Valdimarsdottir UA, Thornorsteinsdottir Thorn K, Lund SH, Tomasson G, Nyberg U, Asgeirsdottir TL, Hauksdottir A (2018) The association between different traumatic life events and suicidality. Eur J Psychotraumatol 9(1):1510279. https://doi.org/10.1080/20008198.2018.1510279

28. Mahoney JJ 3rd, Thompson-Lake DG, Cooper K, Verrico CD, Newton TF, De La Garza R 2nd (2015) A comparison of impulsivity, depressive symptoms, lifetime stress and sensation seeking in healthy controls versus participants with cocaine or methamphetamine use disorders. J Psychopharmacol 29(1):50-56. https://doi. org/10.1177/0269881114560182

29. Cohen S, Kamarck T, Mermelstein R (1983) A global measure of perceived stress. J Health Soc Behav 24(4):385-396

30. Weiss DS (2007) The impact of event scale: revised. In: Wilson JP, Tang CS (eds) Cross-cultural assessment of psychological trauma and PTSD. Springer, pp 219-238

31. Carver CS (1997) You want to measure coping but your protocol's too long: consider the brief COPE. Int J Behav Med 4(1):92-100. https://doi.org/10.1207/s15327558ijbm0401_6

32. Scrignaro M, Barni S, Magrin ME (2011) The combined contribution of social support and coping strategies in predicting posttraumatic growth: a longitudinal study on cancer patients. Psychooncology 20(8):823-831. https://doi.org/10.1002/pon.1782

33. Burke NN, Finn DP, McGuire BE, Roche M (2017) Psychological stress in early life as a predisposing factor for the development of chronic pain: clinical and preclinical evidence and neurobiological mechanisms. J Neurosci Res 95(6):1257-1270. https://doi.org/10. 1002/jnr.23802

34. Witek Janusek L, Tell D, Albuquerque K, Mathews HL (2013) Childhood adversity increases vulnerability for behavioral symptoms and immune dysregulation in women with breast cancer. Brain Behav Immun 30 Suppl:S149-S162. https://doi.org/10. 1016/j.bbi.2012.05.014

35. Parker CH, Naliboff BD, Shih W, Presson AP, Videlock EJ, Mayer EA, Chang L (2019) Negative events during adulthood are associated with symptom severity and altered stress response in patients with irritable bowel syndrome. Clin Gastroenterol Hepatol 17(11): 2245-2252. https://doi.org/10.1016/j.cgh.2018.12.029

36. Hirsch JK, Sirois FM (2016) Hope and fatigue in chronic illness: the role of perceived stress. J Health Psychol 21(4):451-456. https://doi.org/10.1177/1359105314527142

37. Campbell R, Tobback E, Delesie L, Vogelaers D, Mariman A, Vansteenkiste M (2017) Basic psychological need experiences, fatigue, and sleep in individuals with unexplained chronic fatigue. Stress Health 33(5):645-655. https://doi.org/10.1002/smi.2751

38. Chen Z, Wang G, Jiang C (2019) Posttraumatic stress symptoms (PTSS) in patients with Cushing's disease before and after surgery: a prospective study. J Clin Neurosci 66:1-6. https://doi.org/10. 1016/j.jocn.2019.05.059

39. Lee SH, Shin HS, Park HY, Kim JL, Lee JJ, Lee H, Won SD, Han W (2019) Depression as a mediator of chronic fatigue and posttraumatic stress symptoms in Middle East respiratory syndrome survivors. Psychiatry Investig 16(1):59-64. https://doi.org/10. 30773/pi.2018.10.22.3

40. Berger A, Mooney K, Banerjee C, Breitbart W, Carpenter KM, Chang Y, Cleeland C, Dest V, D'uBenske LL, Fernandez-Robles MD, Garcia S, Jankowski C, Jatoi A, Kinczewski L, LeBlanc TW, Loggers ET, Mandrell B, McInnes S, Meyer F, Murphy BA, Palesh O, Plaxe SC, Riba MB, Roshal A, Rugo HS, Salvador C, Wagner N, Walter M (2019) National Comprehensive Cancer Network: NCCN clinical practice guidelines in oncology: cancer-related fatigue. Available at www.nccn.org/professional/physician_gls/pdf/ fatigue.pdf

41. Danhauer SC, Addington EL, Cohen L, Sohl SJ, Van Puymbroeck M, Albinati NK, Culos-Reed SN (2019) Yoga for symptom management in oncology: a review of the evidence base and future directions for research. Cancer 125(12):1979-1989. https://doi.org/ 10.1002/cncr.31979

42. Baitar A, Buntinx F, De Burghgraeve T, Deckx L, Schrijvers D, Wildiers H, van den Akker M (2018) The influence of coping strategies on subsequent well-being in older patients with cancer: a comparison with 2 control groups. Psycho-oncology 27(3):864 870. https://doi.org/10.1002/pon.4587

43. van Muijen P, Schellart AJM, Duijts SFA, van der Beek AJ (2019) The mediating role of coping between self-reported health complaints and functional limitations, self-assessed work ability and work status of long-term sick-listed cancer survivors. Eur J Cancer Care 28(1):e12928. https://doi.org/10.1111/ecc.12928

44. Picariello F, Moss-Morris R, Macdougall IC, Chilcot J (2018) 'It's when you're not doing too much you feel tired': a qualitative exploration of fatigue in end-stage kidney disease. Br J Health Psychol 23(2):311-333. https://doi.org/10.1111/bjhp.12289

45. Dimsdale JE, Ancoli-Israel S, Elsmore TF, Gruen W (2003) Taking fatigue seriously: I. variations in fatigue sampled repeatedly in healthy controls. J Med Eng Technol 27(5):218-222. https://doi. org $/ 10.1080 / 0309190031000075354$

46. Cooklin AR, Giallo R, Rose N (2012) Parental fatigue and parenting practices during early childhood: an Australian community survey. Child Care Health Dev 38(5):654-664. https://doi.org/10. 1111/j.1365-2214.2011.01333.x

47. Tibubos AN, Ernst M, Brahler E, Fischbeck S, Hinz A, Blettner M, Zeissig SR, Weyer V, Imruck BH, Binder H, Beutel ME (2019) Fatigue in survivors of malignant melanoma and its determinants: a register-based cohort study. Support Care Cancer 27(8):28092818. https://doi.org/10.1007/s00520-018-4587-1

48. Holland DP, Schluter DK, Young CA, Mills RJ, Rog DJ, Ford HL, Orchard K, TONiC study group (2019) Use of coping strategies in multiple sclerosis: association with demographic and diseaserelated characteristics. Mult Scler Relat Disord 27:214-222. https://doi.org/10.1016/j.msard.2018.10.016

49. Bussell VA, Naus MJ (2010) A longitudinal investigation of coping and posttraumatic growth in breast cancer survivors. J Psychosoc Oncol 28(1):61-78. https://doi.org/10.1080/07347330903438958

50. Otsuka Y, Sasaki T, Iwasaki K, Mori I (2009) Working hours, coping skills, and psychological health in Japanese daytime workers. Ind Health 47(1):22-32. https://doi.org/10.2486/indhealth.47.22

51. Melton LM (2016) If I don't laugh, I'll cry: exploring humor coping in breast cancer. J Psychosoc Oncol 34(6):530-541. https://doi.org/ 10.1080/07347332.2016.1233926

52. Jiang T, Li H, Hou Y (2019) Cultural differences in humor perception, usage, and implications. Front Psychol 10:123. https://doi.org/ 10.3389/fpsyg.2019.00123

53. Peteet JR, Balboni MJ (2013) Spirituality and religion in oncology. CA Cancer J Clin 63(4):280-289. https://doi.org/10.3322/caac. 21187

54. Lewis S, Salins N, Rao MR, Kadam A (2014) Spiritual well-being and its influence on fatigue in patients undergoing active cancer directed treatment: a correlational study. J Cancer Res Ther 10(3): 676-680. https://doi.org/10.4103/0973-1482.138125

55. Olvera Alvarez HA, Kubzansky LD, Campen MJ, Slavich GM (2018) Early life stress, air pollution, inflammation, and disease: an integrative review and immunologic model of socialenvironmental adversity and lifespan health. Neurosci Biobehav Rev 92:226-242. https://doi.org/10.1016/j.neubiorev.2018.06.002

Publisher's note Springer Nature remains neutral with regard to jurisdictional claims in published maps and institutional affiliations. 